\title{
Electronic quantization in dielectric nanolaminates
}

\author{
T.Willemsen, ${ }^{a, b,{ }^{*}}$ P.Geerke, ${ }^{b}$ M.Jupé, ${ }^{b}$ L.Gallais, ${ }^{c}$ D.Ristau ${ }^{\mathrm{a}, \mathrm{b}}$ \\ ${ }^{a}$ Institut für Quantenoptik Quest, Leibniz University, Hanover, 30167, Germany \\ ${ }^{b}$ Laser Zentrum Hannover e.V., Hollerithallee 8, 30419, Hanover, Germany \\ ${ }^{c}$ Aix Marseille Université, CNRS, Centrale Marseille, Institut Fresnel, UMR 7249, 13013 Marseille, France
}

\begin{abstract}
The scientific background in the field of the laser induced damage processes in optical coatings has been significantly extended during the last decades. Especially for the ultra-short pulse regime a clear correlation between the electronic material parameters and the laser damage threshold could be demonstrated. In the present study, the quantization in nanolaminates is investigated to gain a deeper insight into the behavior of the blue shift of the bandgap in specific coating materials as well as to find approximations for the effective mass of the electrons. The theoretical predictions are correlated to the measurements.
\end{abstract}

Keywords: effective mass, nanolaminate, electronic confinement, ternary composite, 1 on 1 LIDT measurements, new materials

\section{Introduction}

During the last decades the correlation between material parameters and optical as well as electronic properties was investigated in detail. Especially on the basis of ternary composites, both, the index of refraction as well as the band gap energy can be tuned in range between the values of the pure materials [1]. These mixtures were applied in different concepts to improve the laser damage thresholds of optical coating systems. In particular, a stabilization of the laser power handling capability can be achieved by applying gradual interfaces in Rugate filters [2] as well as by employing the RISED concept for Ultra Short Pulse (USP) optics [3]. Also, the damage process in the USP-regime was demonstrated to be dependent on the electronic properties of the involved dielectric materials [4]. In previous investigations [1-4] the content of silica was varied in ternary compounds with different high refractive index materials. In these experiments, an increase of the silica content directly induced a blue shift of the band gap energy and gain of the LIDT-value of the compound material. This general trend could be demonstrated for a broad spectrum ternary oxide compounds consisting of different materials as well as for many pure oxide materials. Often, the ternary composites are manufactured by IBS-Co-sputtering processes where the ternary compound is generated by simultaneous sputtering from two targets. In this approach, the shift of the gap results from the overlap of the wave functions of the different atomic species forming the compound. As an alternative to the co-deposited layers with homogeneously distributed atoms, nanolaminates can be considered for a study of the electronic properties of mixed material phases. If the dimension of the nanolaminate structure is in the range of few nanometers, the bounding leads to the generation of new electronic states [5]. This concept is mainly applied in the quantum well structures or in superlattices which are implemented in semiconductor components like diode lasers or LEDs [6]. In the application of optical components the concept of nanolaminates was used in previous investigations [7]. However the structural sizes of the nano layers were not chosen small enough to generate new electronic states in the structure. Usually, these structures were used for the investigation of mechanical hardness or interface effects [8].

Nowadays the state of the art coating technology achieves accuracies of thin film thickness in subnanometer range [9], which is essential for high end optical components. The Ion Beam Sputtering (IBS) technique was established for the manufacturing of the superior optical quality and the highest accuracy with respect to the film thickness. In contrast to the epitaxial growth, which is used for the manufacturing of semiconductor quantum well structures, IBS produces amorphous layers characterized by a high density and a very low surface roughness. As a consequence of the amorphous structure, the gap of the electronic structure is much more complex than the structure of single crystalline layers. Nevertheless, the quantization of the electronic states should not be limited to single crystalline materials, and therefore, amorphous dielectrics can be applied for the manufacturing of specific nanolaminate structures.

In this study, nanolaminates were manufactured and investigated. The structural sizes of the wells are in the range between $0.5 \mathrm{~nm}$ and $20 \mathrm{~nm}$. The shift of the band gap is measured, and the correlation to the theoretical analysis allows to fit the electronic parameters of the quantum well material. These parameters are applied for the calculation of the single shot LIDT, which are correlated to the measurement results of the structures.

Laser-Induced Damage in Optical Materials 2016, edited by Gregory J. Exarhos, Vitaly E. Gruzdev,

Joseph A. Menapace, Detlev Ristau, MJ Soileau, Proc. of SPIE Vol. 10014, 100140C

(C) 2016 SPIE - CCC code: 0277-786X/16/\$18 - doi: 10.1117/12.2244828

Proc. of SPIE Vol. 10014 100140C-1 


\section{Theory}

The theoretical description has to be divided into two parts to describe the quantization in the two dimensional electron bounding on the one hand and the resulting damage threshold on the other hand. Both theories are well established, and the following description is focused on the main aspects.

The electronic states in a two dimensional structure can be calculated employing solutions of the Schrödinger equation for a potential well which is usually called quantum well structure. Due to the discrete location of the energy levels in sub $\mathrm{nm}$ potential wells, the depth of the potential well has to be included in the modelling of the excitation processes for example considering $\mathrm{HfO}_{2}$ quantum well structures in a Silica matrix. Consequently, the model of limited barriers has to be applied assuming potential walls of equal heights for electrons and holes with respect to the Fermi-level in neutral solids [10]. The effective mass of electrons and holes are fit parameters, while the width of the potential well L is set constant for each calculation. The resulting Eigen-values for the energy are given by solving the transcendent equations

$$
\mathrm{k} \tan \left(\mathrm{k} \frac{\mathrm{L}}{2}\right)=\alpha \quad \text { and } \quad-\mathrm{k} \cot \left(\mathrm{k} \frac{\mathrm{L}}{2}\right)=\alpha
$$

With

$$
\alpha=\frac{1}{\hbar} \sqrt{2 \mathrm{~m}_{\mathrm{e}^{-}, \mathrm{h}^{+}}^{*}\left(\mathrm{E}_{0}-\mathrm{E}_{\mathrm{N}}\right)} \quad \text { and } \quad \mathrm{k}=\frac{1}{\hbar} \sqrt{2 \mathrm{~m}_{\mathrm{e}^{-}, \mathrm{h}^{+}}^{*} \mathrm{E}_{\mathrm{N}}}
$$

Whereby $\hbar$ describes the - Planck quantum, $\mathrm{m}_{\mathrm{e}^{-}, \mathrm{h}^{+}}^{*}$-the effective mass of electrons and holes, $\mathrm{E}_{0}$ denotes the depth of the quantum well and $E_{N}$ the energy of the states, respectively. Equation (1) was solved numerically, and the values are discussed in section results.

For the second branch, the calculation of the laser induced damage threshold in the USP-regime, the model of the density of the quasi free electrons can be applied. In this model, damage in the dielectric material is linked to a critical level $\rho_{\text {crit }}$ of electrons in the conduction band as a starting condition for irreversible damage. The level $\rho_{\text {crit }}$ is given by [11]:

$$
\rho_{\text {crit }}=\frac{\varepsilon_{0} \mathrm{~m}_{\text {eff }} \omega_{0}^{2}}{\mathrm{e}^{2}}=\frac{4 \pi^{2} \mathrm{c}^{2} \varepsilon_{0}}{\mathrm{e}^{2}} \frac{\mathrm{m}_{\text {eff }}}{\lambda^{2}}
$$

Here $m_{\text {eff }}$ refers to the effective mass of an electron, e to the elementary charge, $\omega_{0}$ to the angular frequency, $\mathrm{c}$ to the speed of light, $\varepsilon_{0}$ to the vacuum permittivity, and $\lambda$ to the wavelength, respectively.

The photon energy of the laser $\left(\lambda_{0}=1030 \mathrm{~nm}\right.$; pulse duration $\left.\tau_{\mathrm{p}}=850 \mathrm{fs}\right)$ which is used for the upcoming LIDT tests $\left(\mathrm{E}_{\text {photon }}=1.20 \mathrm{eV}\right)$ is much smaller than the band gap of the dielectric materials $\mathrm{E}\left(E_{\mathrm{Gap}}^{\mathrm{HfO}_{2}}=5.4 \mathrm{eV}\right)$ and therefore, the excitation can be only driven by a nonlinear processes. The excitation is initialized by multiphoton-ionization which generates the necessary electron densities in the range of $10^{18}-10^{19} \mathrm{e}^{-} / \mathrm{cm}^{3}$ for the subsequent avalanche ionization process which both compete with relaxation processes in the solid. Consequently, the electron excitation rate $\partial \rho / \partial \mathrm{t}$ can be described in a first order approximation by the rate equation, considering the intensity $\mathrm{I}(\mathrm{t})$ of the laser beam and the electron density $\rho(t)$ :

$$
\frac{\partial \rho(\mathrm{t})}{\partial \mathrm{t}}=\mathrm{W}_{\mathrm{Pi}}(\mathrm{I}(\mathrm{t}))+\mathrm{W}_{\mathrm{Av}}(\mathrm{I}(\mathrm{t}), \rho(\mathrm{t}))-\mathrm{W}_{\text {rel }}(\rho, \mathrm{t})
$$

For the calculation of the rates different models are available. The photoionization rate $\mathrm{W}_{\mathrm{Pi}}$ is predominantly determined by the Keldysh theory [12]. In this investigation the complete solution including tunnel and multiphoton-ionization is 
used. The avalanche ionization rate $\mathrm{W}_{\mathrm{Av}}$ is calculated by the Multiple Rate Equation (MRE) -model [13], and the relaxation rate $\mathrm{W}_{\text {rel }}$ is implemented by applying a characteristic relaxation time $\tau_{\text {rel }}$ with $W_{r e l}=\frac{\rho}{\tau_{r e l}}$. A detailed description of the theoretical treatment can be found in [3]. In this attempt, the MRE-model delivers a precise energy distribution of the electrons in the conduction band, and consequently, the energy density stored in a system can be calculated by accumulation of the single electron energies according to the equation:

$$
\mathrm{E}_{\text {total }}=\sum_{\mathrm{i}=0}^{\mathrm{k}}(\mathrm{m}+\mathrm{n}) \mathrm{h} v \rho_{\mathrm{i}}
$$

Here, $\mathrm{m}$ denotes the order of the multi photon ionization, $\mathrm{n}$ the energy level in the conduction band, and $\mathrm{k}$ the highest level in the conduction band which is defined by the threshold level for the avalanche ionization. According to the measurement of the laser research center of the Vilnius University, the damage in tantala applying ultra short laser pulses can result from melting of the material after the interaction of the laser beam with matter [14]. To model the melting of the material, the heat capacity is assumed to be constant with temperature $c_{\mathrm{v}}=0.737 \mathrm{~J} / \mathrm{gK}$ and the mass density is 2.5 $\mathrm{g} / \mathrm{cm}^{3}$. The critical temperature is defined by the melting temperature of $1713^{\circ} \mathrm{C}$. These approximations are applicable, because the deposited energy increases rapidly with an increasing electron density in the conduction band. The calculation is based on the excitation process in silica, which is not identical to the excitation in the quantum well structure, because the electronic barriers in the quantum well are reduced with respect to the electronic structure. The total energy is given by the sum of the energy, which is necessary to excite the electron into the band, and the potential energy inside the conduction band, respectively. Nevertheless, the rapid excitation leads to a very similar behavior for the calculated damage threshold independent of modelling a critical electron density or a critical temperature. Figure 1 displays the development of the maximum electron density, and the accumulated energy per volume in the irradiated area. The calculated damage thresholds using a critical electron density are comparable to values gained with the critical temperature method. In detail, for the electronic damage the LIDT results to $3.50 \mathrm{~J} / \mathrm{cm}^{2}$ and for thermal case $3.51 \mathrm{~J} / \mathrm{cm}^{2}$ can be deduced. This difference cannot be clearly distinguished by damage LIDT measurements.

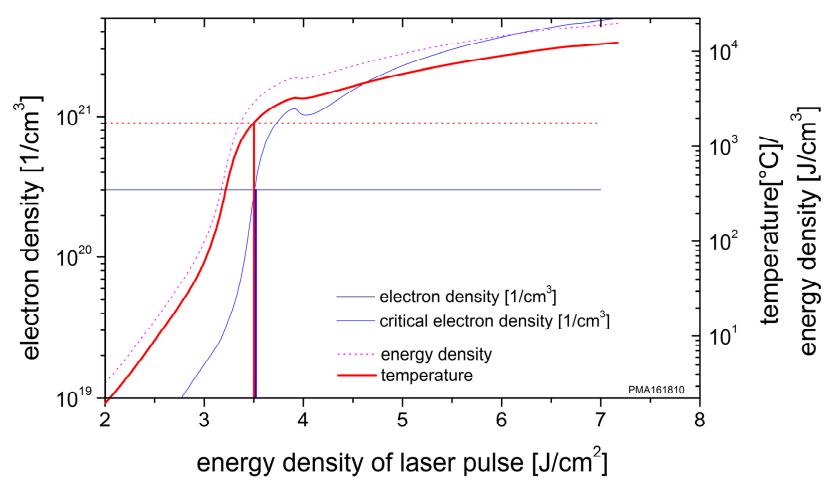

Figure 1: Temperature and electron density in $\mathrm{Si}_{2}$. The density functions were calculated applying a laser wavelength of $1030 \mathrm{~nm}$ and a pulse duration of $850 \mathrm{fs}$.

For the calculation of damage in the QW-structures, the model has to be marginally modified respecting the structure of the material. As mentioned, the excitation process of the electrons is located in the quantum well structure, but with respect to the film thicknesses of a few nm or below and the high melting temperature of Hafnia, melting effects are assumed to occur in the silica matrix.

\section{Deposition Process and LIDT Measurements}

An Ion Beam Sputtering (IBS) process is employed to manufacture the dielectric nanolaminates. In order to achieve sufficient precision in film thickness, the coating plant is equipped with a highly developed broad band optical monitoring system (BBM) [9] which controls in-situ the thickness of each layer. The BBM-system is equipped with a conventional halogen lamp as light source, which is installed below the substrate mount, and a fiber optic CCD spectrometer (Avantes) covering a wavelength range from $420 \mathrm{~nm}$ up to $1050 \mathrm{~nm}$. A schematic drawing of the setup is presented in the attachment. The ion beam is extracted from a Xenon/Argon gas plasma by a three grid system (Veeco 6 $\mathrm{cm}$, beam current $150 \mathrm{~mA}$, acceleration voltage $1500 \mathrm{~V}$ ) and is directed on a flip target assembly. As target materials, Silicon for the low refractive index and Hafnium for the high refractive index were selected, respectively. The layers are 
deposited in a reactive oxygen atmosphere to achieve a balanced stoichiometry. $\mathrm{HfO}_{2}$ layers, characterized by a refractive index of 1.99 at a central wavelength of $\lambda_{0}=800 \mathrm{~nm}$, are sputtered with an average rate of $0.045 \mathrm{~nm} / \mathrm{s}$ and $\mathrm{SiO}_{2}\left(\mathrm{n}_{\mathrm{L}} \approx 1.50\right.$ at $\left.\lambda_{0}=800 \mathrm{~nm}\right)$ layers with $0.185 \mathrm{~nm} / \mathrm{s}$, respectively. The $\mathrm{HfO}_{2} / \mathrm{SiO}_{2}$ layer stacks were deposited on $1 \mathrm{~mm}$ thick fused silica substrates (1 inch diameter) of standard quality and are characterized ex-situ. The optical properties of the deposited samples were derived transmittance and reflectance spectra measured by spectrophotometers. (Perkin Elmer; Lambda 1050 for a wavelength range between $190-2000 \mathrm{~nm}$ and a VUV spectrometer [15] for a wavelength range between 120 and $230 \mathrm{~nm}$ ). On the basis of the obtained absorption coefficient $\alpha(\omega)$ a Tauc approximation is applied to determine the optical gap. In detail, by plotting the square root of the product of the absorption coefficient $\alpha(\omega)$ and the frequency of light $\omega$ versus the photon energy, the optical gap is defined as the intersection point with the photon energy at an absorption coefficient of zero [8]. In addition, the determined optical gaps by Tauc procedure are compared to optical gap values by calculating the Urbach-energy [16]. The recorded spectra of the nanolaminates are approximated as single layers to ascertain the index of refraction $\mathrm{n}(\omega)$ precisely by the Sellmeier Model and for a quantitative comparison to dielectric ternary $\mathrm{HfO}_{2}-\mathrm{SiO}_{2}$ composites.

For further investigations the samples were tested in respect to their single pulse laser induced damage threshold at the Institute Fresnel. For this purpose the samples are irradiated under an angle of incidence of $45^{\circ}$ applying single laser pulses at a center wavelength of $1030 \mathrm{~nm}$ with a duration of $850 \mathrm{fs}$ FWHM and an effective beam size area of $5400 \mu \mathrm{m}^{2}$ (corresponding to a Gaussian beam diameter of $120 \mu \mathrm{m}$ ). The samples are irradiated by a series of single laser shots with increasing fluence. A DIC-microscope picture is taken in-situ for each site, and LIDT is defined when no modification of the layer are observed. The fluence is given for normal incidence in $\mathrm{J} / \mathrm{cm}^{2}$. Further details on the experiment can be found in Ref. [17]. For the following analysis, the respective LIDT values are normalized to the maximum of the calculated field intensity.

\section{Results}

Table 1 summarizes the properties of manufactured nanolaminates. Five different Hafnium and Silicon oxide stacks $(\mathrm{LH})^{\mathrm{n}} \mathrm{L}$ are manufactured by varying the layer thickness of the high refractive index material (low gap material) down to $0.5 \mathrm{~nm}$ and keeping the thickness of the low refractive index barriers as well as the total design thickness of the samples constant. From each coating run half of the manufactured samples were annealed for 12 hours applying a temperature of $300^{\circ} \mathrm{C}$.

Table 1. Properties of the manufactured nanolaminates

\begin{tabular}{ccccc}
\hline $\begin{array}{c}\text { Layer thickness } \\
\mathrm{HfO}_{2}[\mathrm{~nm}]\end{array}$ & $\begin{array}{c}\text { Layer thickness } \\
\mathrm{SiO}_{2}[\mathrm{~nm}]\end{array}$ & Design & $\begin{array}{c}\text { Total thickness } \\
{[\mathrm{nm}]}\end{array}$ & $\begin{array}{c}\text { Optical gap } \\
\text { (Tauc) [eV] }\end{array}$ \\
\hline 8 & 20 & $(\mathrm{LH})^{13} \mathrm{~L}$ & 384 & 5.64 \\
4 & 20 & $(\mathrm{LH})^{15} \mathrm{~L}$ & 380 & 5.76 \\
2 & 20 & $(\mathrm{LH})^{16} \mathrm{~L}$ & 372 & 5.82 \\
1 & 20 & $(\mathrm{LH})^{32} \mathrm{~L}$ & 394 & 6.25 \\
0.5 & 20 & $(\mathrm{LH})^{35} \mathrm{~L}$ & 377 & 6.91 \\
\hline
\end{tabular}

Coating stacks containing $\mathrm{HfO}_{2}$ nanolaminate layers of $8 \mathrm{~nm}$ thickness exhibit optical gap values similar to single $\mathrm{HfO}_{2}$ layers. Reducing the thicknesses to $2 \mathrm{~nm}$ layers, the optical gap increases by almost $3 \%$ towards the ultra violet spectral range. A clear change in the optical gap is obvious for manufactured samples with index barriers of smaller than $2 \mathrm{~nm}$. For instance, the optical gap blue shift of almost $18 \%$ is observed for $\mathrm{HfO}_{2}$ barriers of $0.5 \mathrm{~nm}$. This shift indicates a change in the electron confinement for $\mathrm{HfO}_{2}$ layers smaller than $2 \mathrm{~nm}$ as it is depicted in the transmittance measurements in figure 2a). 


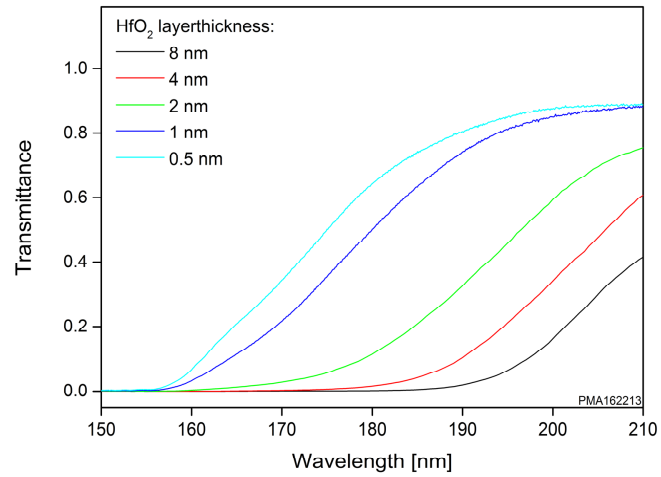

a) Transmittance spectra for different nanolaminates applying high resolution spectrophotometry

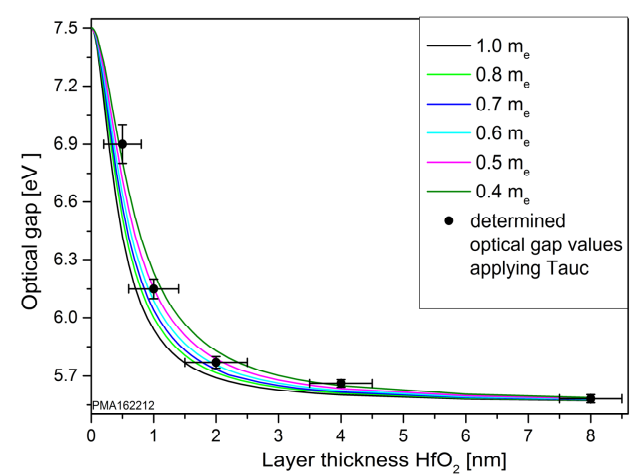

b) Determined optical gap values are correlated to calculated different effective mass $\mathrm{m}_{\mathrm{e}}$.

Figure 2. Spectrophotometric results of the nanolaminates in comparison to theoretical predictions

Solving the Schrödinger equation by estimating a finite potential well of Silica of $7.5 \mathrm{eV}$, the curve progression is depending on the values of the effective mass $m_{e}$ in the valance band and the holes $m_{h}$ in the conduction band as well as the free carriers $m_{p}$ in the conduction band. Figure $2 b$ ) displays several theoretical predictions for different values of $m_{e}$ while keeping the mass of the free carrier $\mathrm{m}_{\mathrm{p}}$ constant. A good correlation between the prediction and the determined optical gap values of the manufactured samples is reached. Taking into account a deposition error of two atomic layers $(0.5 \mathrm{~nm})$ and an error for the optical gap smaller than $0.1 \mathrm{eV}$, an effective electron mass $\mathrm{m}_{\mathrm{e}}$ between $0.4 \mathrm{~m}_{\mathrm{e}}$ and $0.6 \mathrm{~m}_{\mathrm{e}}$ can be assumed.

In the following the LIDT of manufactured nanolaminates in contrast to the optical gap is investigated. A calculated improvement of $30 \%$ of the LIDT value at a center wavelength of $1030 \mathrm{~nm}$ and a pulse width of $850 \mathrm{fs}$ occurs for an increase of the optical gap from $6.2 \mathrm{eV}$ to $6.4 \mathrm{eV}$. The corresponding results of the LIDT measurements are plotted in figure 3a). Three different data sets are considered: Two sets are evaluated by determining the optical gap with the Tauc formalism for the samples with (green data points) and without (red data points) annealing procedure after the deposition. The third one is extracted by ascertaining the optical gap applying Urbach formalism (blue data points). In addition two calculated plots are presented applying the critical temperature model and second the critical electron density model. Both calculations result in a negligible difference in the curve progression (See end of section 2).

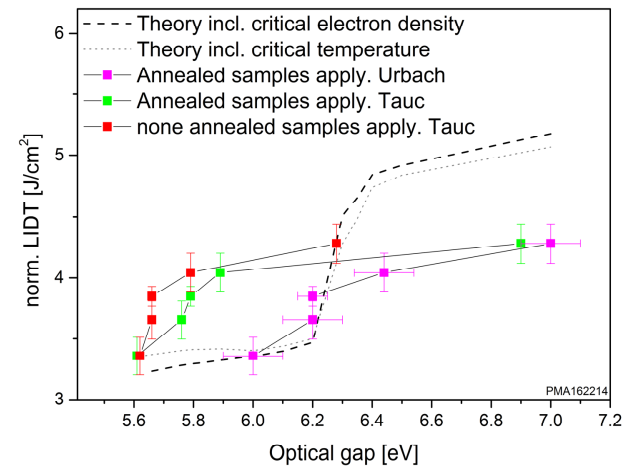

a) Comparison of theoretical and experimental damage behavior.

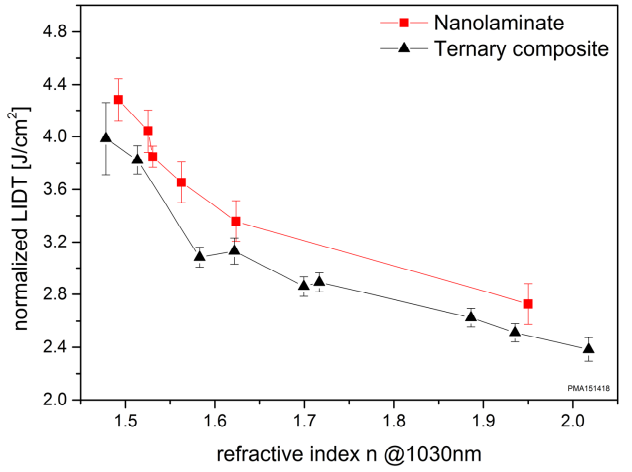

b) Comparison of LIDT values for nanolaminates and ternary composites of $\left(\mathrm{HfO}_{2}-\mathrm{SiO}_{2}\right)$.

Figure 3. LIDT results of the nanolaminates in comparison to theoretical predictions and manufactured ternary composites of $\mathrm{HfO}_{2}-\mathrm{SiO}_{2}$.

Comparing the experimental results to the theoretical prediction, similar trends can be observed. However, the annealed samples evaluated with the Tauc procedure are shifted by approximately $15 \mathrm{~nm}$ to the infra-red spectral range and are suffering from a maximum value which is $20 \%$ lower than the measured laser induced damage threshold. Possible deposition errors of $0.5 \mathrm{~nm}$ in every layer can sum up to a slight shift of the optical gap towards the infra-red spectral wavelength range. In addition the comparison between identical samples evaluated by Tauc and by Urbach formalism 
indicates that both formalisms are influenced by intermedia states in the electron confinement. Thereby the qualitative characteristic of differently weighted absorption coefficient curves can indicate influences by intermedia states, as depicted in fig. 4).

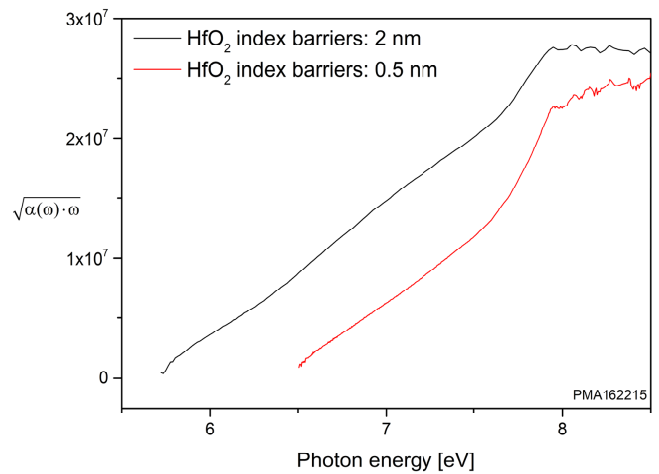

Figure 4. Weighted absorption coefficient plotted against the photon energy. Different characteristics can indicate an influence of intermedia states

Furthermore, the quality of the used substrates and implemented foreign particles due to target motion and different sputtered species are not respected in the theoretical predictions and will additionally lead to lower LIDT results of the higher resistance materials (fig. 5). Also a simple model is used that described well the trends but can not reproduce accurately the results. Nevertheless, both methods deliver an approximation which allows to correlate the results with the theoretical predictions.
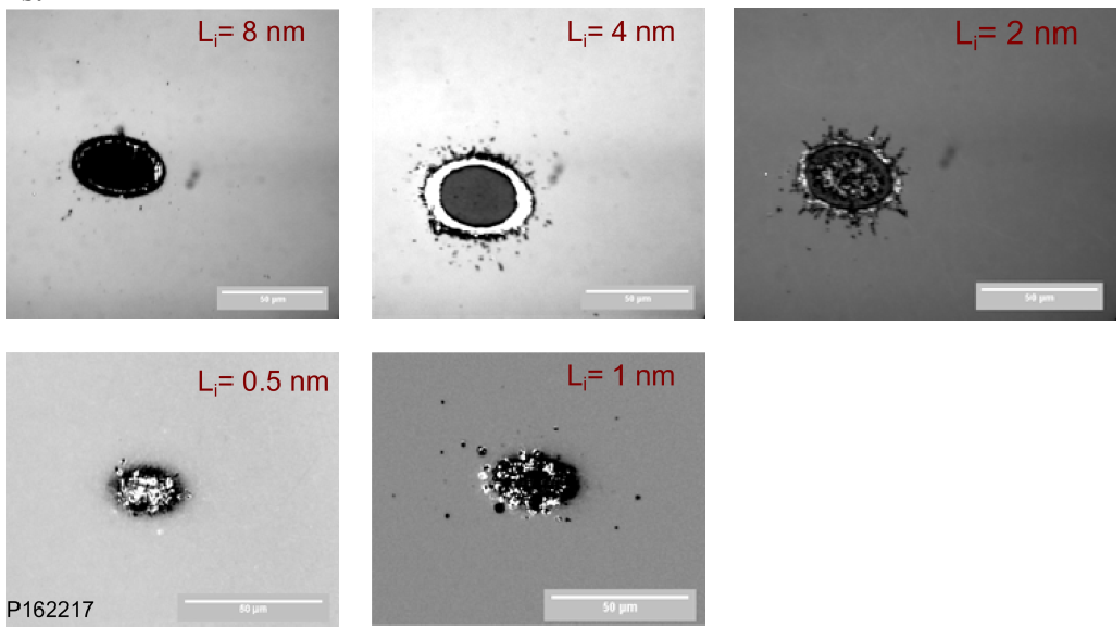

Figure 5. In-situ LIDT DIC-microscope pictures. Different morphologies are obvious depending on the thickness of the index barrier $\mathrm{L}_{\mathrm{i}}$

Finally, the nanolaminates were compared to dielectric ternary composite material of $\mathrm{HfO}_{2}-\mathrm{SiO}_{2}$ with varying content applying IBS-Co-Sputter technique (Fig. 3b). The LIDT of the manufactured ternary composites were determined under the same measurement conditions. The LIDT values of both, the nanolaminates (red data points) and the composites (black data points) were plotted against the refractive index and show similar behavior, qualitatively. With a reduced fraction of $\mathrm{HfO}_{2}$ as well as a smaller width of the quantum well, the LIDT increases, whereas the absolute value of the threshold is higher for the nanolaminate structure with respective refractive indices. Consequently, this indicates that the electron confinement is changed in the manufactured nanolaminates. 


\section{Conclusion}

This paper reports on the possibility to change the electron confinement of dielectric samples by reducing the layer thickness of the high refractive index material $\left(\mathrm{HfO}_{2}\right)$ in a conventional layer stack. Different Hafnium and Silicon oxide coating stacks are manufactured by varying the layer thickness of the high refractive index material (low gap material) down to $0.5 \mathrm{~nm}$ and keeping the thickness of the low refractive index barriers constant. By comparing the resulting shift of the optical gap to the finite quantum well theory, a method is presented to determine the electronic confinement parameters of the material. In order to validate the calculated laser induced damage thresholds applying the critical electron density and the critical temperature, the manufactured samples are irradiated by $850 \mathrm{fs}$ pulses. Beside a general shift, the experimental values show a similar curve progression. Finally, the comparison of LIDT thresholds between manufactured ternary composites of $\mathrm{HfO}_{2}-\mathrm{SiO}_{2}$ and nanolaminates are in good agreement. In future studies it has to be proven that a single dielectric layer of a few $\mathrm{nm}$ builds up as a separate thin film layer when it is embedded in two thicker layers of a low refractive index material.

\section{Acknowledgements}

The authors thank the Ministry for Science and Culture of Lower Saxony and the Volkswagen Stiftung for the financial support of the research project "Hybride Numerische Optik" (contract no. ZN361).

\section{REFERENCES}

[1] Melninkaitis, T. Tolenis, L. Mažulè, J. Mirauskas, V. Sirutkaitis, B. Mangote, X. Fu, M. Zerrad, L. Gallais, M. Commandré, S. Kičas, and R. Drazdys, "Characterization of zirconia- and niobia-silica mixture coatings produced by ion-beam sputtering," Appl. Opt. 50, C188-C196 (2011).

[2] M. Lappschies, B. Görtz, and D. Ristau, "Application of optical broadband monitoring to quasi-rugate filters by ion-beam sputtering," Appl. Opt. 45, 1502-1506 (2006).

[3] M. Jupé, M. Lappschies, L. Jensen, K. Starke, and D. Ristau. "Improvement in laser irradiation resistance of fs- dielectric optics using silica mixtures," Proc. of SPIE 6403 (2007).

[4] M. Jupé, M. Mende, C. Kolleck, D. Ristau, L. Gallais and B. Mangote "Measurement and calculation of ternary oxide mixtures for thin films for ultra short pulse laser optics," Proc. of SPIE 8190 (2011)

[5] H. Kroemer, "Heterostructure bipolar transistors and integrated circuits," Proceedings of the IEEE, 70(1), 13-25 (1982)

[6] H. Jeon, J. Ding, W. Patterson, A. V. Nurmikko, W. Xie, D. C. Grillo, and R. L. Gunshor. "Blue\&green injection laser diodes in (Zn, Cd) Se/ZnSe quantum wells," Applied Physics Letters, 59(27), 3619-3621 (1991)

[7] O. Stenzel, S. Wilbrandt, M. Schürmann, N. Kaiser, H. Ehlers, M. Mende, D. Ristau, S. Bruns, M. Vergöhl, M. Stolze, M. Held, H. Niederwald, T. Koch, W. Riggers, P. Burdack, G. Mark, R. Schäfer, S. Mewes, M. Bischoff, M. Arntzen, F. Eisenkrämer, M. Lappschies, S. Jakobs, S. Koch, and B. Baumgarten, "Tailored Nanocomposite Coatings for Optics," in Optical Interference Coatings, OSA Technical Digest (Optical Society of America, 2010), paper MD2

[8] M. El-Eskandarany, "Mechanical solid state mixing for synthesizing of $\mathrm{SiC} \mathrm{p} / \mathrm{Al}$ nanocomposites," Journal of alloys and Compounds, 279(2), 263-271 (1998)

[9] D. Ristau, H. Ehlers, T. Gross, and M. Lappschies, "Optical broadband monitoring of conventional and ion processes," Applied Optics, 45(7), 1495-1501 (2006)

[10] C. Kittel, "Introduction to solid state" (John Wiley \& Sons, 1966) Chap6.

[11] M. Jupé, L. Jensen, A. Melninkaitis, V. Sirutkaitis, and D. Ristau, "Calculations and experimental demonstration of multi-photon absorption governing fs laser-induced damage in titania," Optics Express, 17(15), 12269-12278 (2009)

[12] L. V. Keldysh. "Diagram technique for nonequilibrium processes," Sov. Phys. JETP, 20(4), 1018-1026 (1965)

[13] B. Rethfeld,. "Unified model for the free-electron avalanche in laser-irradiated dielectrics," Physical Review Letters, $92(18), 187401$ (2004).

[14] N. Šiaulys, L. Gallais, and A. Melninkaitis, "Direct holographic imaging of ultrafast laser damage process in thin films," Opt. Lett. 39, 2164-2167 (2014)

[15] H. Blaschke, J. Kohlhaas, P. Kadkhoda, and D. Ristau: "DUV/VUV spectrophotometry for high precision spectral characterization," Proc. of SPIE, vol. 4932, pp. 536-543, Proceedings of the 34th Annual Symposium on Laser-Induced Damage in Optical Materials, (2003)

[16] F. Urbach: "The long-wavelength edge of photographic sensitivity and of the electronic absorption of solids," Physical Review, $92(5), 1324$ (1953)

[17] B. Mangote, L. Gallais, M. Zerrad, F. Lemarchand, L. H. Gao, M. Commandré, and M. Lequime, "A high accuracy femto-/picosecond laser damage test facility dedicated to the study of optical thin films," Rev. Sci. Instrum. 83, 013109 (2012) 


\section{Attachment}

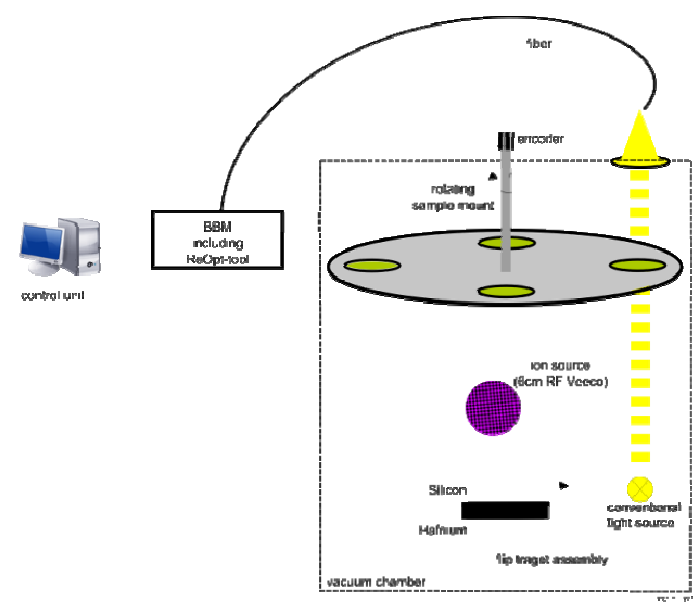

Figure A1. Schematic drawing of the machine.

Proc. of SPIE Vol. 10014 100140C-8

Downloaded From: http://proceedings.spiedigitallibrary.org/ on 07/20/2017 Terms of Use: http://spiedigitallibrary.org/ss/termsofuse.aspx 